\title{
A GTP-state specific cyclic peptide inhibitor of the GTPase Gas
}

\author{
Shizhong A. Dai ${ }^{1,2 \dagger}$, Qi Hu${ }^{1,2 \dagger}$, Rong Gao ${ }^{3 \dagger}$, Andre Lazar ${ }^{1,4 \dagger}$, Ziyang Zhang ${ }^{1,2}$, Mark von \\ Zastrow $^{1,4}$, Hiroaki Suga ${ }^{3 *}$, Kevan M. Shokat ${ }^{1,2^{*}}$
}

${ }^{1}$ Department of Cellular and Molecular Pharmacology, University of California San Francisco, San Francisco, CA, 94158, USA

${ }^{2}$ Howard Hughes Medical Institute

${ }^{3}$ Department of Chemistry, Graduate School of Science, The University of Tokyo, 7-3-1 Hongo, Bunkyo-ku, Tokyo 113-0033, Japan

USA

*Correspondence to: hsuga@chem.s.u-tokyo.ac.jp, kevan.shokat@ucsf.edu

$\dagger$ These authors contributed equally. 


\section{Abstract:}

The G protein-coupled receptor (GPCR) cascade leading to production of the second messenger cAMP is replete with pharmacologically targetable receptors and enzymes with the exception of the stimulatory G protein $\alpha$ subunit, Gas. GTPases remain largely undruggable given the difficulty of displacing high affinity guanine nucleotides and the lack of other drug binding sites. We explored a chemical library of $10^{12}$ cyclic peptides in order to expand the chemical search for inhibitors of this enzyme class. We identified a macrocyclic peptide, GsIN-1, that antagonizes the GTP-bound active state of Gas with high G protein specificity and nucleotide-binding-state selectivity. A $1.57 \AA$ co-crystal structure reveals that GsIN-1 binds to a novel switch II / $\alpha 3$ pocket in Gas and directly blocks adenylyl cyclase activation. GsIN-1 inhibits isoproterenol-stimulated Gas activation through binding to the crystallographically defined pocket. The discovery of GsIN1 provides path for the development of state-dependent GTPase inhibitors. 


\section{Introduction}

The family of human GTPases represents a vast but largely untapped source of pharmacological targets. They serve as key molecular switches that control cell growth and proliferation through cycling between tightly regulated ON/OFF states. The role of specific GTPase family members across diverse human diseases have been widely established by cancer genome sequencing (e.g., $K R A S, G N A S, G N A Q$ and others) and by familial studies in neurodegenerative disease (e.g. LRRK2, RAB39B) (1-4). Despite the widespread recognition of these disease target relationships, only very recently has the first drug targeting a GTPase K-Ras (G12C) achieved clinical proof of principle $(5,6)$. The covalent somatic cysteine mutant-specific nature of the K-Ras $(\mathrm{G} 12 \mathrm{C})$ drugs has opened the potential for targeting a GTPase for the first time.

Several non-covalent GTPase binders have been reported, but their target scope has been limited. The macrocyclic natural product YM-254890 is a cyclic depsipeptide targeting Gaq (the $\alpha$-subunit of the hetero-trimeric $\mathrm{G}$ protein $\mathrm{Gq}$ ) with high specificity and potency (7). Despite the highly conserved nature of $\mathrm{G}$ proteins and the recent chemical tractability of fully synthetic YM254890 , efforts to use this natural macrocycle as a scaffold from which to discover inhibitors of other $\mathrm{G}$ proteins (Gas, Gai) have not been successful (8-10), likely because of a limited number of diverse YM-254890 analogs. Notably, both YM-254890 and the current covalent inhibitors of K-Ras (G12C) bind only to the inactive GDP-bound states of their target GTPases (5-7), but several disease-relevant GTPases, including K-Ras, Gas and Gaq, are locked in a constitutively activated state in cancer cells $(1,2,11)$. We therefore reasoned that since the active state of GTPases is their GTP rather than GDP state, we should preferentially target GTP state of GTPases. Gas, also called GNAS, is the most frequently mutated Ga protein in cancer and is currently considered undruggable (2). We hypothesized that by screening an ultra-large chemical library of 
cyclic peptides against the GTP state of Gas we might be able to discover a Gas specific inhibitor that discriminates between the active and inactive states of Gas and potentially open the remainder of the GTPase family to pharmacological studies.

The Random nonstandard Peptide Integrated Discovery (RaPID) system (12) is an in vitro display system which merges the flexibility of an in vitro translation system (FIT) (13-16) with mRNA display, enabling the screening of exceptionally large macrocyclic peptide libraries (> $10^{12}$ ) against challenging targets (17). Here we report the discovery by the RaPID system of GsIN1, a macrocyclic peptide that is the first known cell permeable selective binder of the active conformation of Gas, with high selectivity over other G protein subfamilies and nucleotide binding states.

\section{Results}

\section{Selection of cyclic peptides that bind to the active state of Gas}

Unbiased screening hits emerging from the RaPID cyclic peptide selection process against Gas could theoretically bind anywhere on the surface of the protein and so might or might not perturb its function. To bias hits toward function-perturbing binders, we took advantage of the fact that when Gas switches from the GDP-bound inactive state to the GTP-bound active state significant conformational changes occur on one face of Gas, comprising the so-called switch I, II and III regions (18), which are known to bind effector proteins such as adenylyl cyclases. We reasoned that performing both a positive selection against the GTP-bound state of Gas and a negative selection against the GDP-bound state would enrich for binders to the switch regions, and that these binders would be likely to disrupt Gas function.

We performed the positive selection with wild-type Gas (WT Gas) bound to the nonhydrolyzable GTP analogue GppNHp (5'-guanylyl imidodiphosphate) and the negative selection 
against GDP-bound WT Gas (Fig. 1). Starting from a cDNA library, each round of selection included PCR amplification of the cDNA library, in vitro transcription into an mRNA library, ligation with a puromycin linker, and translation to generate a peptide library covalently conjugated with its encoding mRNA library (Fig. S1A). The library encoded peptides contain an N-chloroacetyl-D-tyrosine at the N-terminus, followed by 8-12 random proteinogenic amino acids encoded by NNK codons, a cysteine residue and a GSGSGS linker (Fig. 1B). Cyclization occurs spontaneously between the chloroacetyl group and the thiol group of the cysteine residue. The peptide-ligated mRNA library was further reverse-transcribed into a cDNA-mRNA-peptide library, subjected to negative selection against GDP-bound Gas, then followed by positive selection against GppNHp-bound Gas (Fig. S1A).

After four rounds of selection (R1-R4), cyclic peptide binders for the GppNHp-bound Gas were enriched (Fig. 1A) and further identified by next generation sequencing (NGS). The sequences of the top 20 hits were aligned and shown in Fig. 1B. Peptides selective for GppNHpbound Gas from the R4 pool were identified and quantified by comparison selection (Fig. S1B and Fig. 1C). Nine of the top twenty hits, those with more than 100-fold selectivity for GppNHpbound over GDP-bound Gas (indicated by red triangles in Fig. 1B) were chosen for further analysis.

\section{The cyclic peptide GsIN-1 blocks Gos-mediated adenylyl cyclase activation}

To evaluate the cyclic peptide hits without the appended DNA/mRNA duplex, residues from the N-chloroacetyl-D-tyrosine to the glycine (after the anchor cysteine residue) of the selected peptides were synthesized using solid phase peptide synthesis followed by in situ cyclization. We refer to these synthesized peptides with a "GN" preceding their ranking number. In order to determine whether these cyclic peptides inhibited Gas activity, we assayed the ability of Gas to 
activate its downstream effector adenylyl cyclase (AC). We evaluated the inhibitory effect of our top hits on Gas-mediated adenylyl cyclase activation by measuring production of cAMP in a reconstituted Gas activity assay. Among these nine cyclic peptides, GN13 showed the greatest inhibition, with an $\mathrm{IC}_{50}$ of $4.15 \pm 1.13 \mu \mathrm{M}$ (Fig. 1D and E). We renamed this novel Gas inhibitor

as GsIN-1. GsIN-1 did not inhibit forskolin-mediated, Gas-independent adenylyl cyclase activation (Fig. S2), suggesting a Gas-dependent mechanism of inhibition.

\section{The crystal structure of the GppNHp-bound Gas in complex with GsIN-1}

To elucidate how the cyclic peptide GsIN-1 binds to Gas and inhibits Gas-mediated adenylyl cyclase activation, we solved a co-crystal structure of the Gas/GppNHp/GsIN-1 complex. The structure was determined by molecular replacement and refined to $1.57 \AA$ (table S1). The overall structure is shown in Fig. 2A. GsIN-1 assumes a highly ordered structure through extensive intramolecular and intermolecular hydrogen bonding networks with three well-defined water molecules (Fig. S3, S4A and S4B). One molecule of GsIN-1 binds to a pocket between switch II and the $\alpha 3$ helix of GppNHp-bound Gas through hydrogen bonding and hydrophobic interactions from the $\varepsilon$-amino group of K274 in Gas; the indole ring of residue W9 in GsIN-1 donates a hydrogen bond to the side chain of E268 in Gas; the main chain carbonyl oxygens of residues V5, W9 and T11, and the main chain amide of W9 in GsIN-1 form five hydrogen bonds with residues N279, R280, R231, R232, and S275 on switch II and the $\alpha 3$ helix in Gas (Fig. 2B). The side chains of residues I8 and W9 in GsIN-1 dock into two hydrophobic pockets on Gas (Fig. 2C). The structural analysis reveals that residue W9 in GsIN-1 is the most important residue mediating the interactions between GsIN-1 and Gas, contributing three hydrogen bonds as well as hydrophobic interactions. 
The Gas/GppNHp/GsIN-1 complex structure strongly resembles the Gas/GTP $\gamma \mathrm{S}$ structure (PDB code: 1AZT) (19), suggesting that GsIN-1 recognizes the active state conformation and does not induce significant conformational change upon binding (Fig. 2D). We also aligned our structure with the structure of inactive GDP-bound Gas (chain I in PDB 6EG8) (20). In comparison with our structure, the N-terminus of switch II in the GDP-bound Gas is unstructured and close to the $\alpha 3$ helix, with nearly half of the GsIN-1/Gas interface disrupted (Fig. 2E and F). In particular, R232 of switch II (shown in space filling) is in a restrictive position relative to I8 of GsIN-1. The nucleotide-state specificity of GsIN-1 binding is therefore determined by the conformation of switch II.

The natural product YM-254890 inhibits GDP dissociation from Gaq by directly binding to the hydrophobic cleft between two interdomain linkers connecting the GTPase and helical domains of the Gaq (Fig. S5) (7). By contrast, GsIN-1 occupies a distinct pocket in Gas and inhibits Gas as a direct protein-protein interaction inhibitor. Comparison between the

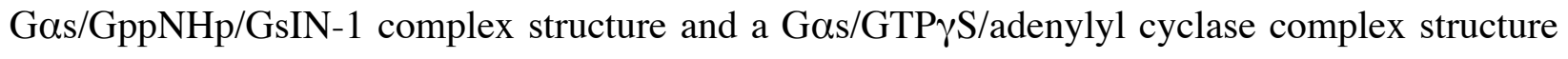
(PDB code: 1AZS) suggested that GsIN-1 directly occludes the hydrophobic interaction between Gas and adenylyl cyclase (21), which accounts for the inhibitory effect of GsIN-1 (Fig. 2G to I). To further confirm the mechanism of inhibition, we tested the physical interaction between active Gas and adenylyl cyclase in the presence of GsIN-1 using a fluorescence resonance energy transfer (FRET) assay (Fig. S6A). GsIN-1 showed strong, dose-dependent inhibition of the interaction 20 between Gas and adenylyl cyclase (Fig. S6B).

\section{The cyclic peptide GsIN-1 is able to penetrate HeLa cell membrane}

With a potent Gas inhibitor, GsIN-1, functioning in vitro, we next asked whether GsIN-1 could penetrate cell membranes, as peptide-based chemical probes often suffer from poor cell 
permeability. Several G protein-specific linear peptides exhibit in vitro activities but have no reported cellular efficacy, likely due to their low cell permeability (22-27). In order to quantitatively evaluate the cell permeability of GsIN-1, we used a recently developed HaloTagbased assay known as a chloroalkane penetration assay (CAPA) (Fig. 3A) (28). HeLa cells stably expressing HaloTag localized to the mitochondrial outer membrane were pulsed with chloroalkane-tagged molecules (ct-molecule), washed, chased with chloroalkane-tagged TAMRA fluorophore (ct-TAMRA), and finally analyzed by flow cytometry. A lower ct-TAMRA fluorescent signal indicates competition from a higher cytosolic concentration of ct-molecule. We replaced the glutamate residue at the 3rd position of GsIN-1 with a glutamine residue to simplify chemical synthesis while maintaining the hydrogen binding interaction with K274 in Gas. The carboxyl terminus of GsIN-1 (Gly14) was conjugated with a chloroalkane tag to make ct-GsIN-1 (E3Q) (Fig. S7A). ct-GsIN-1 (E3Q) showed similar biochemical activity to unmodified GsIN-1, indicating that the chloroalkane modification and E3Q mutation did not impact the proper conformation and ligand-protein interaction of GsIN-1 (Fig. S8). We used a cell-permeable chloroalkane-tagged tryptophan (ct-W, Fig. S7B) as a positive control for ct-TAMRA competition. In comparison with ct-W, ct-GsIN-1 (E3Q) showed lower but acceptable cell permeability a CP50 value of $12.2 \pm 0.4 \mu \mathrm{M}$ (Fig. 3B).

\section{GsIN-1 inhibits isoproterenol-stimulated Gas activation in HEK293 cells}

We next tested GsIN-1 in HEK 293 cells that endogenously express the $\beta 2$-adrenoceptors ( $\beta 2$ ARs) at low levels, making them a useful model for studying Gas cellular signaling in the presence of upstream receptors. We examined the $\beta 2-\mathrm{AR}-\mathrm{G} \alpha \mathrm{s}-\mathrm{cAMP}$ response elicited by the $\beta 2-\mathrm{AR}$ agonist isoproterenol at a saturating concentration $(1 \mu \mathrm{M})$ by monitoring live cAMP concentration with an established luminescence-based reporter (pGlo-20F). Both GsIN-1 and ct-GsIN-1 (E3Q) 
were able to inhibit isoproterenol-mediated cAMP production in a dose dependent manner (Fig. 3 $\mathrm{C}$ and D). For GsIN-1, the $\mathrm{IC}_{50}$ value in this cellular assay $(3.39 \pm 0.33 \mu \mathrm{M})$ and that in the previous biochemical assay $(4.15 \pm 1.13 \mu \mathrm{M})$ were very consistent, suggesting that GsIN-1 acts in cells by specifically inhibiting Gas.

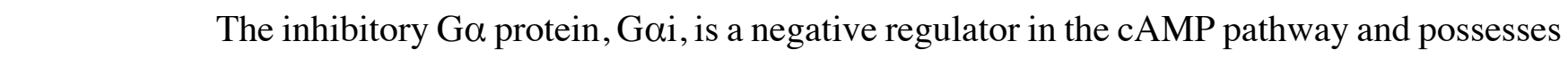
a structure similar to that of Gas. To assess whether GsIN-1 was capable of discriminating between

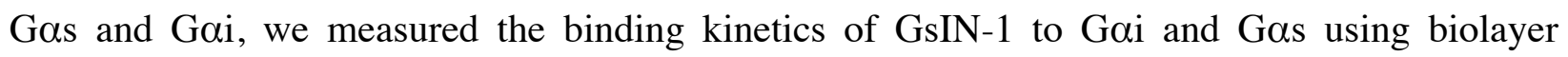
interferometry (BLI). We found that GsIN-1 binds to immobilized GppNHp-bound WT Gas with a $K_{d}$ value of $0.19 \pm 0.02 \mu \mathrm{M}$ (Fig. S9A). By contrast, GsIN-1 had no detectable binding affinity to GDP-bound WT Gas, GDP-bound WT Gail or GppNHp-bound WT Gai1 (Fig. S9 B to D), confirming the selectivity of GsIN-1 for the active state of Gas.

\section{A drug-resistant Gas mutant blocks GsIN-1 inhibition}

To further assess the cellular specificity of GsIN-1, we designed a GsIN-1-resistant Gas mutant. We examined the structures of the Gas/GsIN-1 complex and Gas/AC complex and noticed that serine 275 in Gas makes close contact with GsIN-1 but this residue does not contact adenylyl cyclase (Fig. 4, A and B). We reasoned that mutating this serine residue to a bulky residue should create a drug-resistant Gas mutant that blocks the GsIN-1-Gas interaction but would have little effect on adenylyl cyclase activation. Indeed, Gas S275L mutant maintained a similar level of biochemical activity but was resistant to GsIN-1 inhibition. (Fig. 4 C to E). We tested GsIN-1 in GNAS-knockout (GNAS-KO) HEK 293 cells that do not express endogenous Gas protein (29). GsIN-1 was able to inhibit isoproterenol-mediated cAMP production in a dose-dependent manner when WT Gas was reintroduced into the GNAS KO cells by transient transfection. By contrast, when the drug resistant mutant Gas S275L was reintroduced into GNAS KO cells, the inhibitory 
effect of GsIN-1 was abolished (Fig. 4F and G), providing further evidence that the observed pharmacological activity is due to GsIN-1 binding to the switch region of Gas.

\section{Conclusion}

GPCRs and $\mathrm{G}$ proteins comprise the largest family of signal transducing proteins in the human

genome. Although approximately $35 \%$ of approved drugs target GPCRs, directly targeting the downstream integrator $\mathrm{G}$ proteins has potentiated a broader efficacy via blocking converged pathways shared by multiple GPCRs $(30,31)$. However, there is a striking absence of drug-like chemical matter that specifically targets the active state of G $\alpha$ proteins in cells. The cyclic peptide inhibitor GsIN-1 recognizes a novel pocket in the GTP-bound Gas where downstream effectors directly engage. Our demonstration of the use of the RaPID cyclic peptide platform through both positive and negative selection steps provides path for discovery of other cell-permeable, classspecific and state-selective inhibitors of the remainder of the GTPase family. 


\section{References and Notes:}

1. I. A. Prior, P. D. Lewis, C. Mattos, A comprehensive survey of Ras mutations in cancer. Cancer Res. 72, 2457-2467 (2012).

2. M. O'Hayre et al., The emerging mutational landscape of G proteins and G-proteincoupled receptors in cancer. Nat. Rev. Cancer. 13, 412-424 (2013).

3. D. R. Alessi, E. Sammler, LRRK2 kinase in Parkinson's disease. Science. 360, 36-37 (2018).

4. G. R. Wilson et al., Mutations in RAB39B cause X-linked intellectual disability and earlyonset Parkinson disease with $\alpha$-synuclein pathology. Am. J. Hum. Genet. 95, 729-735 (2014).

5. J. Canon et al., The clinical KRAS(G12C) inhibitor AMG 510 drives anti-tumour immunity. Nature. 575, 217-223 (2019).

6. J. Hallin et al., The KRASG12C Inhibitor MRTX849 Provides Insight toward Therapeutic Susceptibility of KRAS-Mutant Cancers in Mouse Models and Patients. Cancer Discov. 10, 54-71 (2020).

7. A. Nishimura et al., Structural basis for the specific inhibition of heterotrimeric Gq protein by a small molecule. Proc. Natl. Acad. Sci. U.S.A. 107, 13666-13671 (2010).

8. H. Kaur, P. W. R. Harris, P. J. Little, M. A. Brimble, Total synthesis of the cyclic depsipeptide YM-280193, a platelet aggregation inhibitor. Org. Lett. 17, 492-495 (2015).

9. X.-F. Xiong et al., Total synthesis and structure-activity relationship studies of a series of selective G protein inhibitors. Nat Chem. 8, 1035-1041 (2016). 
10. H. Zhang et al., Structure-Activity Relationship Studies of the Cyclic Depsipeptide Natural Product YM-254890, Targeting the Gq Protein. ChemMedChem. 12, 830-834 (2017).

11. H. Qi et al., Disease-causing mutations in the G protein Gas subvert the roles of GDP and GTP. Cell. 173, 1254-1264 (2018).

12. Y. Yamagishi et al., Natural product-like macrocyclic N-methyl-peptide inhibitors against a ubiquitin ligase uncovered from a ribosome-expressed de novo library. Chem Biol. 18, 1562-70 (2011).

13. H. Murakami et al., A highly flexible tRNA acylation method for non-natural polypeptide synthesis. Nat Methods. 3, 357-9 (2006).

14. H. Murakami et al., A versatile tRNA aminoacylation catalyst based on RNA. Chem Biol. 10, 655-62 (2003).

15. K. Ramaswamy et al., Designer ribozymes: programming the tRNA specificity into flexizyme. J Am Chem Soc. 126, 11454-5 (2004).

16. H. Xiao et al., Structural basis of specific tRNA aminoacylation by a small in vitro selected ribozyme. Nature. 454, 358-61 (2008).

17. T. Passioura, H. Suga, A RaPID way to discover nonstandard macrocyclic peptide modulators of drug targets. Chem. Commun.(Camb.). 53, 1931-1940 (2017).

18. D. G. Lambright, J. P. Noel, H. E. Hamm, P. B. Sigler, Structural determinants for activation of the alpha-subunit of a heterotrimeric G protein. Nature. 369, 621-628 (1994).

19. R. K. Sunahara, J. J. Tesmer, A. G. Gilman, S. R. Sprang, Crystal structure of the adenylyl cyclase activator Gsalpha. Science. 278, 1943-1947 (1997). 
20. X. Liu et al., Structural Insights into the Process of GPCR-G Protein Complex Formation. Cell. 177, 1243-1251 (2019).

$21 \mathrm{~J}$ Tesmer et al., Crystal structure of the catalytic domains of adenylyl cyclase in a complex with Gs $\alpha \cdot G T P \gamma$ S. Science. 278, 1907-1916 (1997).

22. W. W. Ja, R. W. Roberts, In vitro selection of state-specific peptide modulators of G protein signaling using mRNA display. Biochemistry. 43, 9265-9275 (2004).

23. C. A. Johnston et al., Structure of Galpha(i1) bound to a GDP-selective peptide provides insight into guanine nucleotide exchange. Structure. 13, 1069-1080 (2005).

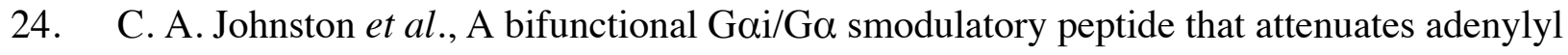
cyclase activity. FEBS Letters. 579, 5746-5750 (2005).

25. R. J. Austin, W. W. Ja, R. W. Roberts, Evolution of class-specific peptides targeting a hot spot of the Galphas subunit. J. Mol. Biol. 377, 1406-1418 (2008).

26. C. A. Johnston et al., Minimal Determinants for Binding Activated G $\alpha$ from the Structure of a Gai1-Peptide Dimer. Biochemistry. 45, 11390-11400 (2006).

27. T. H. Charpentier et al., Potent and Selective Peptide-based Inhibition of the G Protein Gaq. J. Biol. Chem. 291, 25608-25616 (2016).

28. L. Peraro et al., Cell Penetration Profiling Using the Chloroalkane Penetration Assay. J. Am. Chem. Soc. 140, 11360-11369 (2018).

29. W. Stallaert et al., Purinergic receptor transactivation by the $\beta 2$-adrenergic receptor increases intracellular $\mathrm{Ca}^{2+}$ in nonexcitable cells. Mol. Pharmacol. 91, 533-544 (2017).

30. T. M. Bonacci et al., Differential targeting of $\mathrm{G} \beta \gamma$-subunit signaling with small molecules. Science. 312, 443-446 (2006). 
31. S. Gulati et al., Targeting G protein-coupled receptor signaling at the G protein level with a selective nanobody inhibitor. Nat. Commun. 9, 1-15 (2018).

32. Y. Goto et al., Flexizymes for genetic code reprogramming. Nat Protoc. 6, 779-90 (2011).

33. Z. Otwinowski, W. Minor, Processing of X-ray diffraction data collected in oscillation mode. Meth. Enzymol. 276, 307-326 (1997).

34. P. Evans, Scaling and assessment of data quality. Acta Crystallogr. D Biol. Crystallogr. 62, 72-82 (2006).

35. A. J. McCoy et al., Phaser crystallographic software. J Appl Crystallogr. 40, 658-674 (2007).

36. M. D. Winn et al., Overview of the CCP4 suite and current developments. Acta Crystallogr. D Biol. Crystallogr. 67, 235-242 (2011).

37. P. Emsley, B. Lohkamp, W. G. Scott, K. Cowtan, Features and development of Coot. Acta Crystallogr. D Biol. Crystallogr. 66, 486-501 (2010).

38. P. D. Adams et al., PHENIX: a comprehensive Python-based system for macromolecular structure solution. Acta Crystallogr. D Biol. Crystallogr. 66, 213-221 (2010).

39. J. Morimoto, Y. Hayashi, H. Suga, Discovery of macrocyclic peptides armed with a mechanism-based warhead: isoform-selective inhibition of human deacetylase SIRT2. Angew Chem Int Ed Engl. 51, 3423-7 (2012).

40. T. K. Neklesa et al., Small-molecule hydrophobic tagging-induced degradation of HaloTag fusion proteins. Nat. Chem. Biol. 7, 538-543 (2011). 
Acknowledgments: We would like to thank the staff at A.L.S. beamline 8.2.1. We also would like to thank Drs. Xiaobo Wan and Xi Liu for assistance in X-ray data processing and structure refinement. Funding: This work was supported by the Howard Hughes Medical Institute (K.M.S.); NIH grants R1R01CA244550 (K.M.S.), DA010711, DA012864, and MH120212 (M.v.Z.); Japan Agency for Medical Research and Development (AMED), Platform Project for Supporting Drug Discovery and Life Science Research (Basis for Supporting Innovative Drug Discovery and Life Science Research) under JP19am0101090 (H.S.). S.A.D is a UCSF Discovery fellow and a UCSF Fletcher Jones Fellow.; Q.H. is a Damon Runyon Fellow supported by the Damon Runyon Cancer Research Foundation (DRG-[2229-15]).; Z.Z. is a Damon Runyon Fellow supported by the Damon Runyon Cancer Research Foundation (DRG-[2281-17]). Author contributions: K.M.S., and H.S. conceived the project; S.A.D., Q.H., R.G., K.M.S., and H.S. designed the experiments. R.G. performed cyclic peptide selection using the RaPID system; R.G., S.A.D., and Z.Z. carried out the chemical synthesis of cyclic peptides; S.A.D., and Q.H. performed biochemical characterization of GsIN-1, including protein purification, the adenylyl cyclase activation assay, the FRET assay, the BLI assay; Q.H. crystallized the GsIN-1/Gas complex and determined the structure; S.A.D. performed the CAPA assay; A.L., and S.A.D. performed cellular characterization of GsIN-1 using the pGlo assay; S.A.D., Q.H., and K.M.S wrote the manuscript with the contribution from other authors. Competing interests: The authors declare no competing interests. Data and materials availability: The accession number for the crystal structure of GppNHp-bound GNAS in complex with the cyclic peptide inhibitor GsIN-1 reported in this paper is PDB: 7BPH. All other data are available in the manuscript or the supplementary materials. 
bioRxiv preprint doi: https://doi.org/10.1101/2020.04.25.054080; this version posted April 27, 2020. The copyright holder for this preprint (which

was not certified by peer review) is the author/funder, who has granted bioRxiv a license to display the preprint in perpetuity. It is made available under aCC-BY-NC-ND 4.0 International license.

\section{Supplementary Materials:}

Materials and Methods

Figures S1-S9

Tables S1

5

References (32-40) 
bioRxiv preprint doi: https://doi.org/10.1101/2020.04.25.054080; this version posted April 27, 2020. The copyright holder for this preprint (which

was not certified by peer review) is the author/funder, who has granted bioRxiv a license to display the preprint in perpetuity. It is made available under aCC-BY-NC-ND 4.0 International license.

Figure 1. RaPID selection of active state Gas binding cyclic peptides.

A

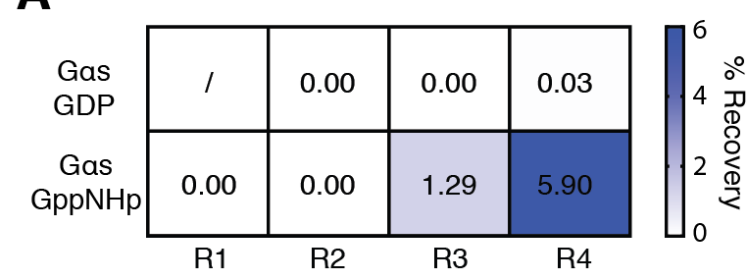

C

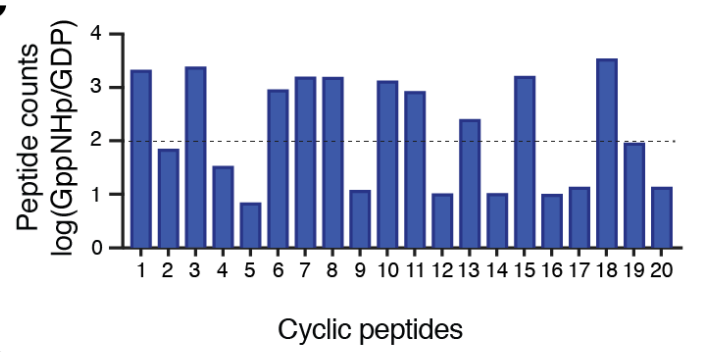

D

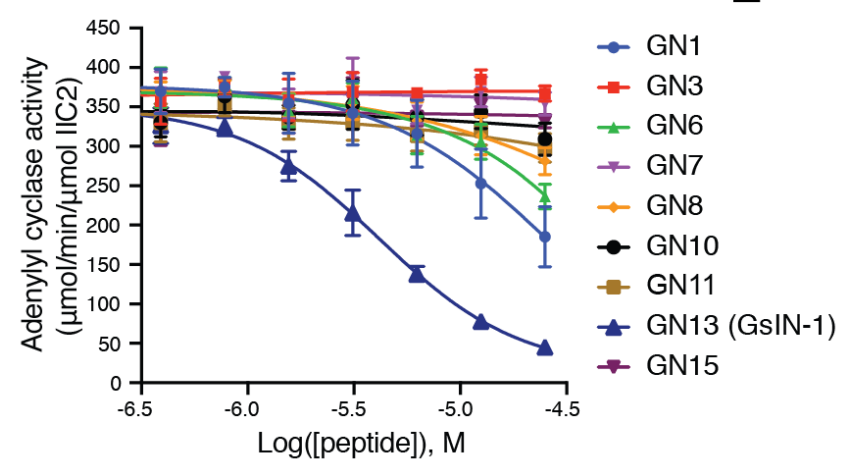

B Ranking 1 Peptide Backbone

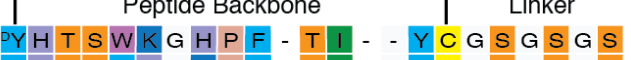
PYHTS FHG KPF - TI - - YCGSGSGS PYH TSWKGHPF - TI - Y YGS - - PYHTSYGG I PF - SI - - YCGSGSGS DYH TS YGG I PF - S I - Y YGS - - DYHT S F Q N R P F - S V - Y Y G S G S G DYHTSWNR KP DYH TSWA Q K P Y - TV - Y YGSGSGS

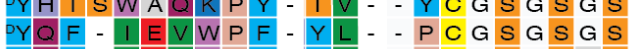
DYQF - I EVWPF - YL - PCGSGSAS DYCF - YEVWWP - YL - PCGSGSGS

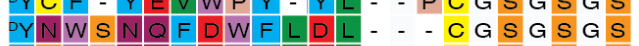
DYSWPNQFDWFIIEL - - CGSGSGS PYH - E Q F D N F C D L - WCGSGSGS DYK YWD - FD NF Q E L - YCGSGSGS DY K L W A N V V F G I Y GA - - C GSGSGS PYOY TY - - P Y L V I DW T C G S G S G DY L T NWG V YWDQV - - WCGSGS G DYDE I WGS LN . . S S SCGSGSGS PYFESV - - - YA I WG TLCGSGSGS

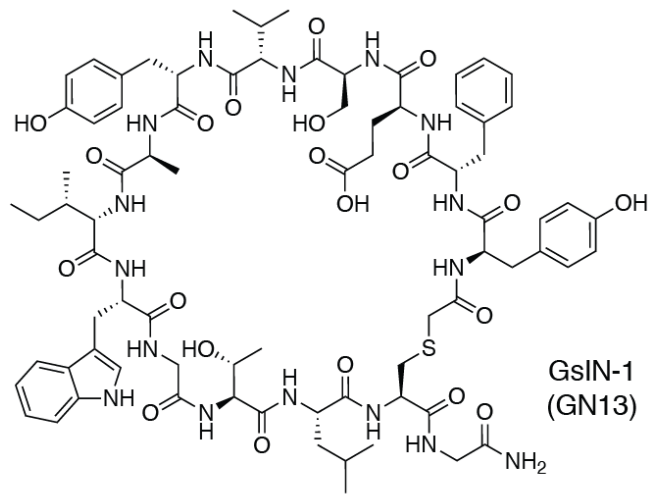


Fig. 1. RaPID selection of active state Gos binding cyclic peptides.

(A) The percentage of enriched peptide-mRNA-cDNA complex in the input library after each selection was quantified by qPCR. Cyclic peptides that bind to GppNHp-bound Gas were enriched through four rounds of RaPID selection. For better enrichment, negative selection was not included in the first round of selection. (B) Sequence alignment of top 20 cyclic peptides from the last round of positive selection. The sulfur bridge cyclizes peptides between D-tyrosine and cysteine. The 18th peptide (asterisk) was not selected because it has the same sequence as the 1st peptide except a Gly to Ala mutation in the linker region. (C) Comparison selection was performed by analyzing peptide-mRNA-cDNA complex binding to GDP-bound or GppNHp-bound Gas-immobilized beads, respectively. A high ratio means a better selectivity against GppNHp-bound Gas. Cyclic peptides with more than 100-fold selectivity are marked with a red triangle in (B) and were selected for solid phase synthesis. (D) Activation of adenylyl cyclase by Gas was inhibited by cyclic peptides in a dose-dependent manner. GppNHp-bound Gas was mixed with various concentrations of cyclic peptides, adenylyl cyclase (VC1/IIC2), and forskolin. After adding ATP, the reaction was carried out at $30{ }^{\circ} \mathrm{C}$ for $10 \mathrm{~min}$. Production of cAMP was evaluated by the LANCE Ultra cAMP kit. The data represent the mean $\pm \mathrm{SE}$ of three independent measurements. (E) Chemical structure of resynthesized cyclic peptide GsIN-1 (GN13). 
bioRxiv preprint doi: https://doi.org/10.1101/2020.04.25.054080; this version posted April 27, 2020. The copyright holder for this preprint (which was not certified by peer review) is the author/funder, who has granted bioRxiv a license to display the preprint in perpetuity. It is made available under aCC-BY-NC-ND 4.0 International license.

Figure 2. Crystal Structure of GppNHp-bound Gas in complex with GsIN-1.

A

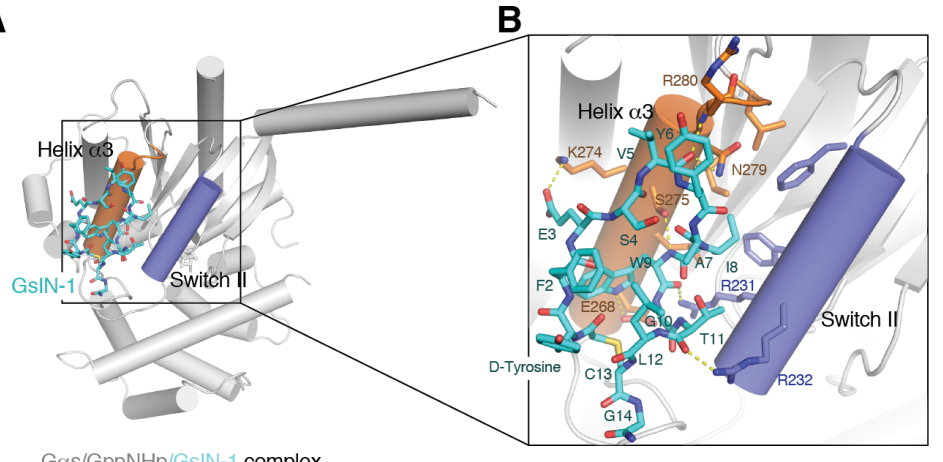

C

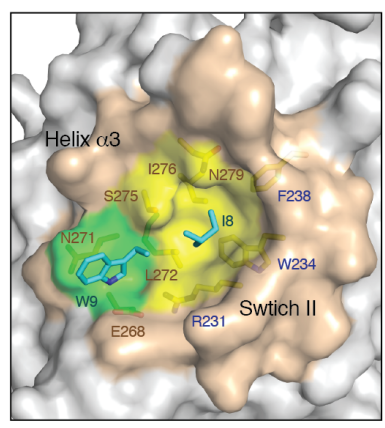

Gas/GppNHp/GsIN-1 complex

D

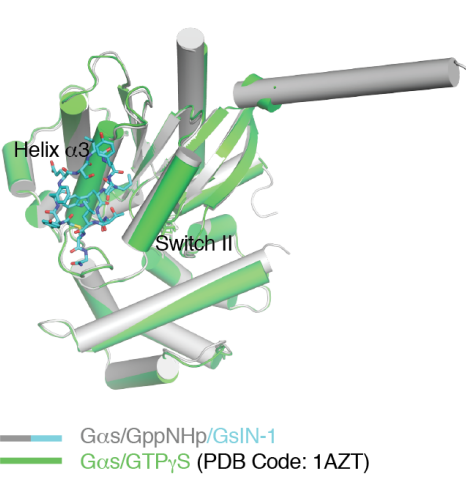

E

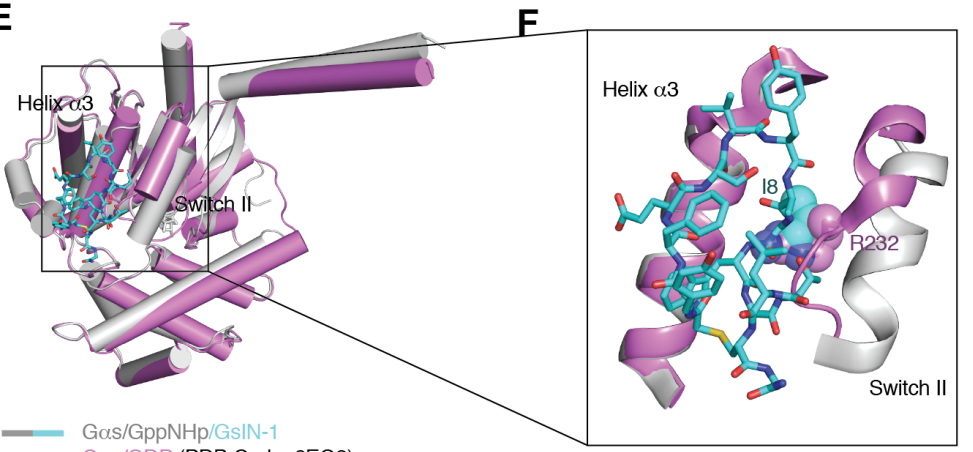

- Gas/GppNHp/GsIN-1

Gas/GDP (PDB Code: 6EG8)

G

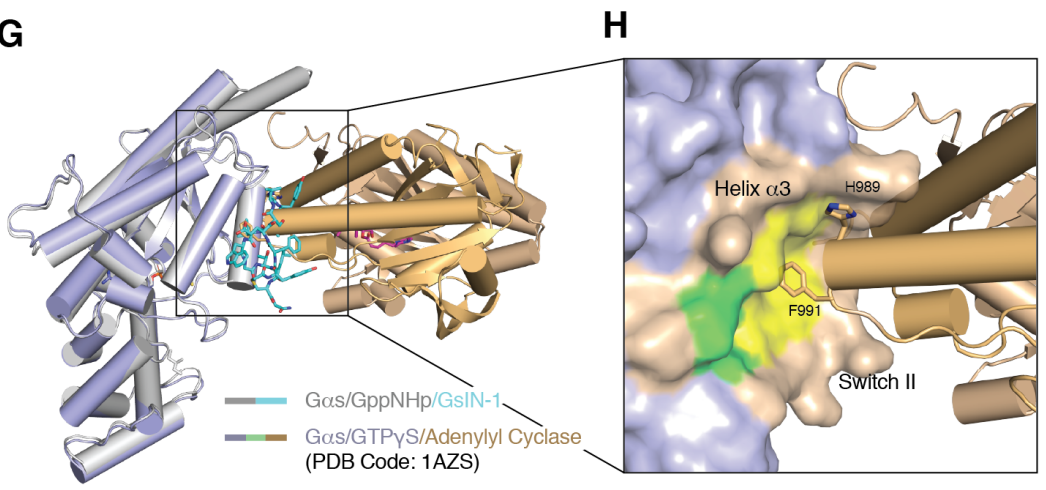

I

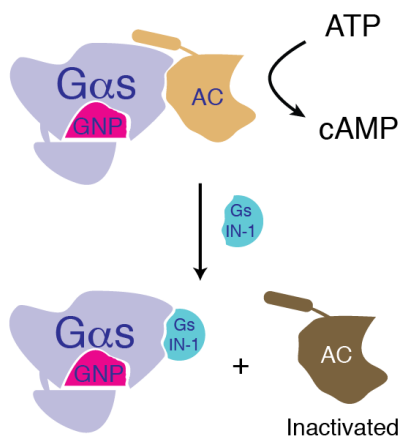


Fig. 2. Crystal Structure of GppNHp-bound Gas in complex with GsIN-1.

(A) Overall structure of the GsIN-1/GppNHp-bound Gas complex. GsIN-1 (cyan) binds in between the switch II region (slate) and the $\alpha 3$ helix (orange). GsIN-1 and GppNHp are shown as sticks. (B) Structural details of the GsIN-1-Gas interaction. Hydrogen bonds are represented by

yellow dashed lines. (C) Close-up view of two hydrophobic pockets (green and yellow) that accommodate the tryptophan and isoleucine side chains of GsIN-1 (cyan). Residues that form those pockets are depicted as stick models and labeled. (D) Alignment of Gas/GsIN-1 complex structure (grey) with the structure of GTP $\gamma \mathrm{S}$-bound Gas (green) (PDB: 1AZT). Root mean square deviation $($ RMSD) $=0.449 \AA$. (E) Alignment of Gas/GsIN-1 complex structure (grey) with the

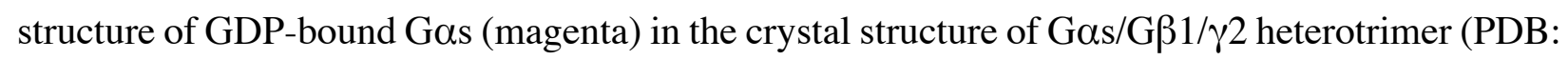
6EG8). (F) Structural basis for nucleotide-state-selective binding of GsIN-1 to Gas. In GDPbound Gas, switch II is partially disordered, which disrupts polar contacts with GsIN-1 and creates extensive steric hindrance. In particular, R232 of switch II (shown in space filling) is in a restrictive position relative to I8 of GsIN-1. (G) Alignment of the Gas/GsIN-1 complex structure (grey) with the structure of GTP $\gamma$ S-bound Gas (light blue)/adenylyl cyclase complex (orange) (PDB: 1AZS). (H) Close-up view of the interaction between GTP $\gamma$ S-bound Gas (light blue) and adenylyl cyclase (light orange). Two hydrophobic pockets in Gas that interact with GsIN-1 are highlighted in green and yellow. I8 of GsIN-1 and H989/F991 of adenylyl cyclase bind to the same hydrophobic pocket (yellow) in Gas. (I) Schematic representation of GsIN-1 inhibiting Gas mediated adenylyl cyclase activation. 
Figure 3. The cell permeable cyclic peptide GsIN-1 inhibits Gos activity in HEK 293 cells

A

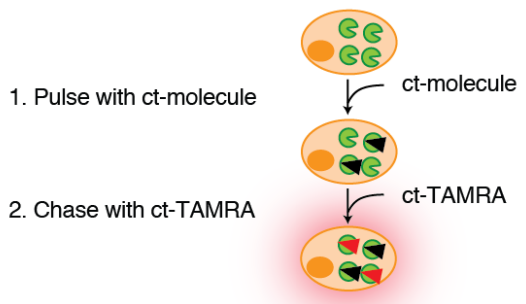

3. Anaylze by Flow cytometry

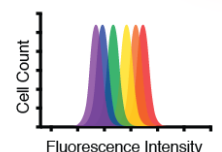

C

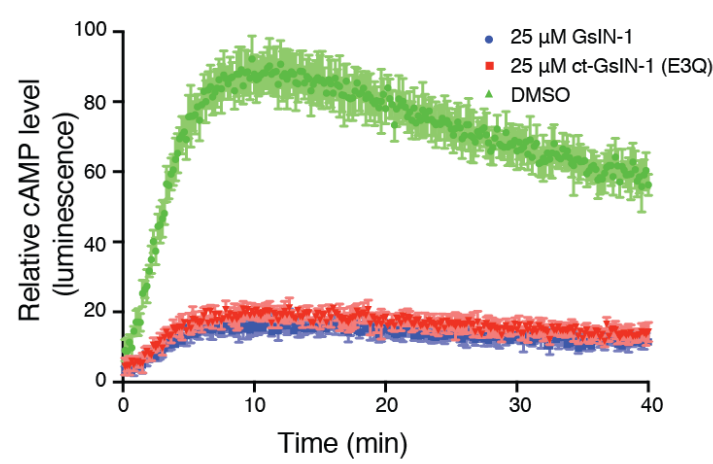

B

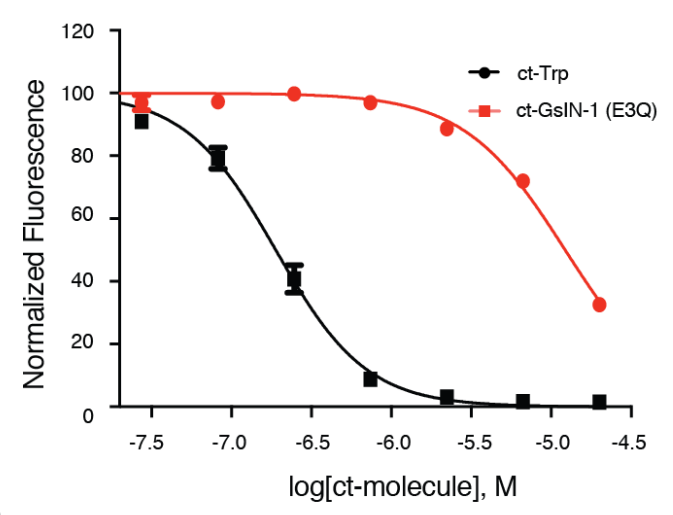

D

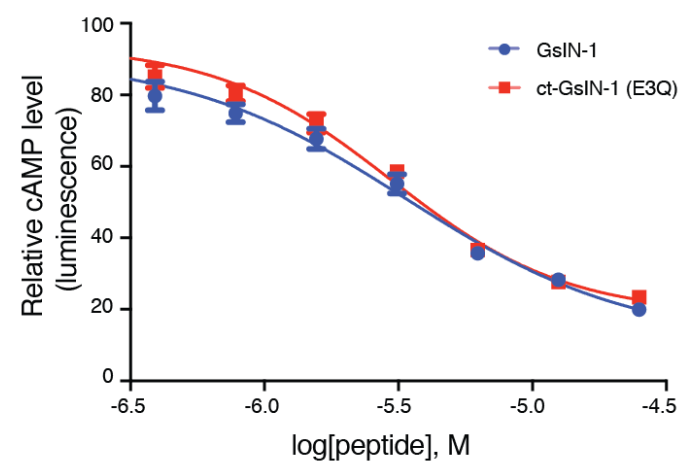


Fig. 3. The cell permeable cyclic peptide GsIN-1 inhibits Gas activity in HEK 293 cells

(A) Schematic representation of the chloroalkane penetration assay (CAPA). HeLa cells stably express GFP-tagged HaloTag on the mitochondrial outer membrane. If the pre-dosed chloroalkane-tagged molecule (ct-molecule) penetrates the cell membrane, it will covalently label HaloTag and block subsequent HaloTag labeling with ct-TAMRA. Intracellular ct-TAMRA fluorescence intensity is inversely related to the amount of cytosolic ct-molecule. (B) CAPA results for ct-W (black) and ct-GsIN-1 (E3Q) (red). Each point is the median ct-TAMRA fluorescence of 10,000 cells. The data were normalized using cells that were only treated with ctTAMRA as $100 \%$ signal and cells that were not treated with any ct-compound as $0 \%$ signal. The data represent the mean \pm SD of three independent measurements. $(\mathbf{C})$ Real-time cAMP levels were measured using the enzyme-based biosensor pGLO-20F (Promega) in response to application of $1 \mu \mathrm{M}$ isoproterenol in HEK 293 cells pretreated for 165 min with DMSO (green), $25 \mu \mathrm{M}$ GsIN1 (blue) or $25 \mu \mathrm{M}$ ct-GsIN-1 (E3Q) (red). The data represent the mean \pm SD of four independent biological replicates. (D) HEK 293 cells were pretreated with various concentrations of GsIN-1 (blue) or ct-GsIN-1(E3Q) (red) for $165 \mathrm{~min}$, and then stimulated with $1 \mu \mathrm{M}$ isoproterenol. Each point is the averaged maximum cAMP signal from one real-time luminescence measurement. The data represent the mean \pm SD of four independent biological replicates. 
Figure 4. The Gas S275L mutation confers resistance to GsIN-1 inhibition.

A

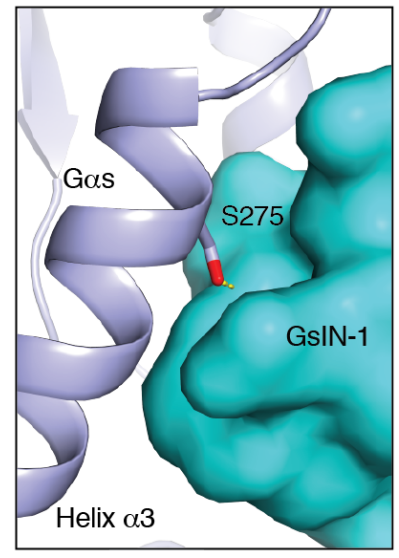

D

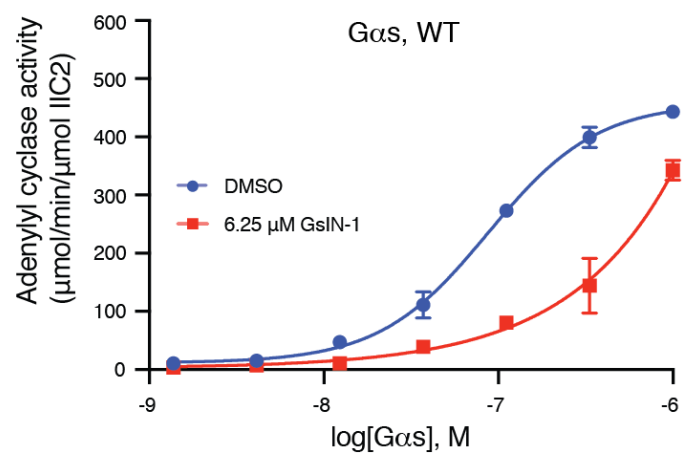

$\mathbf{F}$

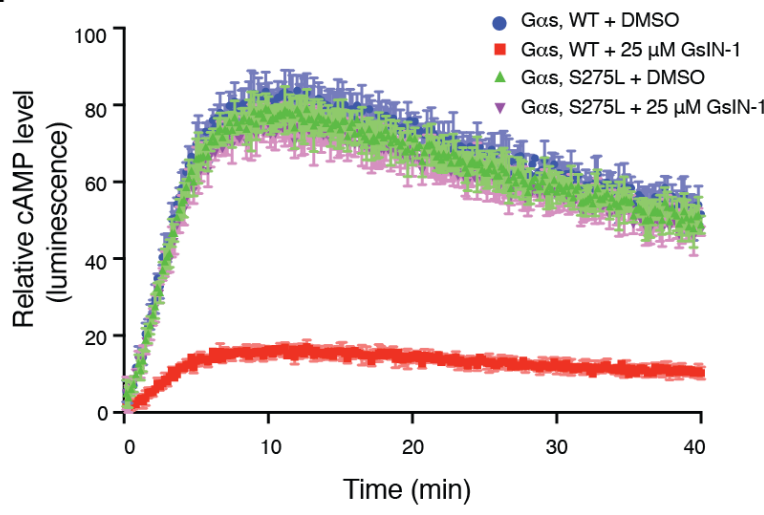

C

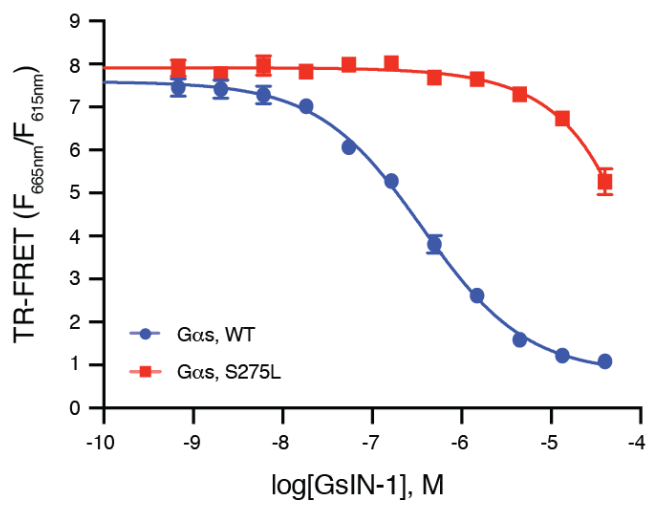

$\mathbf{E}$
G
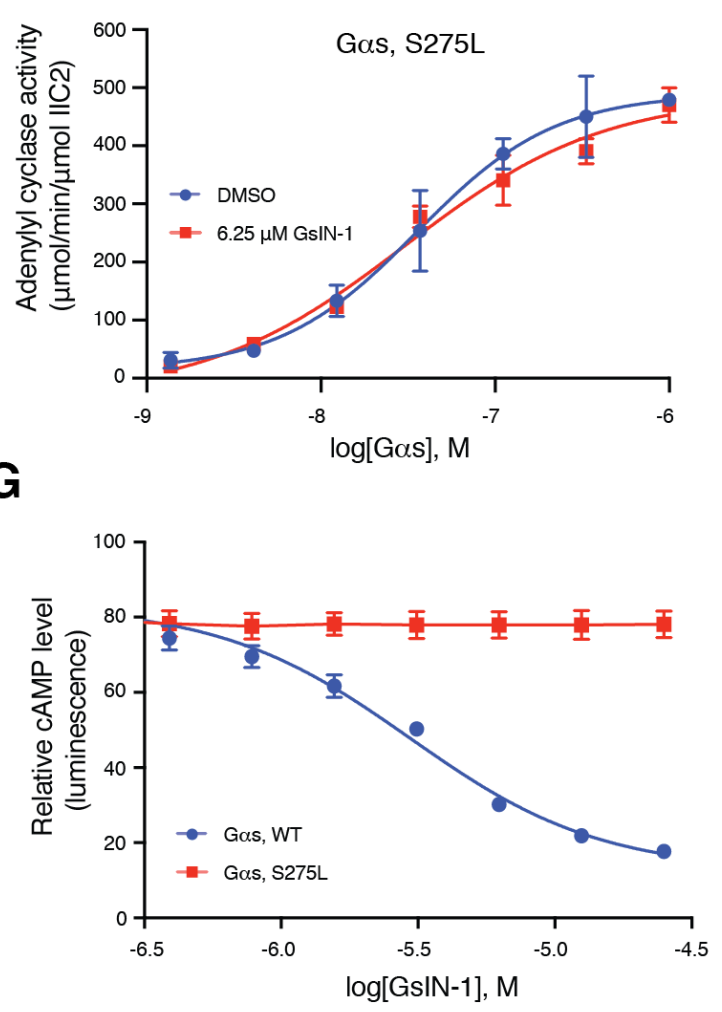
Fig. 4. The Gas S275L mutation confers resistance to GsIN-1 inhibition.

(A and B) Close-up view of the interaction between GsIN-1 (Cyan) and the Gas $\alpha 3$ helix (light blue) in (A) and the interaction between adenylyl cyclase (light orange) and the Gas $\alpha 3$ helix in (B) (PDB: 1AZS). S275 is shown as sticks. Hydrogen bonds are represented by yellow dashed lines. (C) His-tagged adenylyl cyclase was labeled with Tb-cryptate (FRET donor). Biotinylated Gas WT and S275L mutant were labeled with XL665 (FRET acceptor). GsIN-1 inhibited the protein-protein interaction of Gas WT with adenylyl cyclase in a dose-dependent manner (blue). This inhibitory effect was significantly diminished by the S275L mutation (red). The data represent the mean \pm SD of three independent measurements. (D and E) WT Gas and the S275L mutant have comparable biochemical activities in an adenylyl cyclase activation assay in the presence of $\mathrm{G} \beta 1 / \gamma 2$ (blue curves in D and E). $6.25 \mu \mathrm{M}$ of GsIN-1 inhibits adenylyl cyclase activation by Gos WT (red curve in D) but not by Gas S275L (red curve in E). The data represent the mean \pm SD of three independent measurements. (F) GNAS KO HEK 293 cells were transiently transfected with the same amount of Gas WT or Gas S275L constructs. Real-time cAMP levels were measured using the enzyme-based biosensor pGLO-20F (Promega) in response to application of $1 \mu \mathrm{M}$ isoproterenol in the above-mentioned cells pretreated for 165 min with DMSO (blue or green), 25 $\mu \mathrm{M}$ GsIN-1 (red or magenta). The data represent the mean \pm SD of four independent biological replicates. (G) GNAS KO HEK 293 cells were transiently transfected with Gas WT (blue) or S275L mutant (red) constructs and pretreated with various concentrations of GsIN-1 for 165 min, and then stimulated with $1 \mu \mathrm{M}$ isoproterenol. Each point is the averaged maximum cAMP signal from one real-time luminescence measurement. The data represent the mean \pm SD of four independent biological replicates. 